\title{
Laboratory Study on the Propagation of Horizontal Edge Cracks in Rock-like Material
}

\author{
Baoliang Zhang \\ School of Architecture and Civil \\ Engineering \\ Liaocheng University \\ Liaocheng, China \\ zhangbaoiang@lcu.edu.cn \\ Xizhen Sun \\ College of Mining and Safety \\ Engineering \\ Shandong University of Science and \\ Technology \\ Qingdao, China \\ sunxizhen2008@126.com
}

\author{
Baotang Shen \\ College of Mining and Safety \\ Engineering \\ Shandong University of Science and \\ Technology \\ Qingdao, China \\ baotang.shen@csiro.au
}

\author{
Shaojie Chen \\ College of Mining and Safety \\ Engineering \\ Shandong University of Science and \\ Technology \\ Qingdao, China \\ chensj@sdust.edu.cn
}

\begin{abstract}
Rock fracture propagation is a major hazard for mining and tunnel excavation in fractured rock masses or coal seams. This paper describes a study of investigating the mechanisms and pathways of rock fracture under uniaxial compression. A rock-like material which consists of model gypsum, water and diatomaceous earth at a mass ratio of 165:75:2 were used. The uniaxial compression strength of the material decreased with the increase of the length of preexisting horizontal edge crack. During the tests, wing (tensile) cracks were first observed at the tip of the pre-existing horizontal edge crack. This was followed by secondary cracks as the loading increasing. The final failure of the specimens however was dominated by tensile cracks throughout the specimens. Due to the sudden crack initiations in the specimens, the loading stress in the specimen varies changes stepwise and AE energy and amplitude showed abrupt changes when crack initiated. When the crack initiation occurred, the loading stress of the specimens showed a notable retreat in the stress-strain curve, and the recorded $\mathrm{AE}$ energy and amplitude showed a sharp spike. Understanding the horizontal edge crack propagation characteristics and fracture mechanisms is a key step for the prevention and control of floor water inrush in longwall mining panels.
\end{abstract}

Keywords-pre-existing edge crack, rock-like material, fracturing, propagation, uniaxial compression, floor failure

\section{INTRODUCTION}

Rock fracture propagation is one of the key concerns in many rock engineering problems because it could lead to rock mass instability and rapid increase of water inflow. For deep underground coal mines using the longwall mining method, a major safety issue is the water inrush from the aquifers above or below the mining horizon. A longwall mining panel with a typical dimension of $200 \mathrm{~m}$ (width) $\times$ $1000 \mathrm{~m}$ (length) $\times 3 \mathrm{~m}$ (height) can be considered as an open edge crack. Due to its relatively thin height, it may be considered as an open edge crack under the compressive stress (overburden stress). The fracturing processes in the vicinity of the edge crack (or the longwall panel) particularly in the roof and floor are critically important for the safety of mining operation because fracturing can lead to water inrush and dynamic loading on the working face.

Over the past several decades, a large number of studies have been performed to investigate the fracture mechanical behaviors of pre-existing cracks. The law of edge crack propagation under the action of load or heat flow was studied with the means of theoretical analysis and numerical simulation by Zhang et al [1-3].The approximate modified Mohr-Coulomb criterion has been implemented in a fracture mechanics based numerical code FRACOD by Shen et al [4]. The failure mechanical behaviors of different joint angles in rock-coal bodies were studied by Yin et al [5-6]. Zhu et al gave studies on the characteristics and failure mechanism of crack propagation with $\mathrm{AE}$ monitoring system under uniaxial compression tests [7-8]. A deformation analysis model was established between rock matrix and micro-cracks to reflect the initial macroscopic deformation of rock and determine micro-cracks closure stress by Zhang et al [9-11]. The confining pressure of $0,5,10 \mathrm{MPa}$ were separately applied to the rock specimen and the effect on the crack propagation as well as the fracturing mode was analyzed by Liu et al [12]. To observe rock failure process, many engineering researchers have adopted technologies like acoustic emission, high-speed camera and computer tomography (CT) etc. Dang et al, Sun et al and Yun et al developed an image analyzing software to characterize the deformation and failure of rocklike materials with a single pre-existing crack [13-15]. At the same time, many scholars have done a lot of numerical simulation experiments on crack propagation [16-19].

The above achievements have important significance for understanding the mechanism of rock fracture propagation. Meanwhile, due to the aspects of testing material selection, the existing studies often focus on the propagation mechanism of crack coalescence and contained 3D cracks and the studies on horizontal edge crack extension of rock materials are few. This paper explores the propagation characteristics of horizontal edge cracks under compressive loading. Brittle material made of gypsum and water was used to simulate the real rock behavior in the mining environment. 
The results hence will be directly relevant to the seam floor brush.

\section{TESTING MATERIAL AND METHOD}

\section{A. Testing Material}

Although it would be highly desirable to use actual rock from the field, that would involve a lot of difficulties. During specimen preparation, cracks and the vibration from rock cutting would be likely to destroy the specimen. In addition, using field rock would lead to highly variable testing results, making it difficult to observe the mechanisms. For this reason, we used rock-like material to simulate the process of crack propagation. The testing material was a mixture of model gypsum, diatomite and water $\left(a t 30^{\circ} \mathrm{C}\right)$ with a ratio of 165:2:75 by weight. The resulting material had a tensile strength of $2.4 \mathrm{MPa}$. This material could effectively simulate the crack propagation process in brittle rock.

The specimens were cuboids with dimensions $100 \mathrm{~mm}(\mathrm{~L})$ $\times 100 \mathrm{~mm}(\mathrm{~W}) \times 200 \mathrm{~mm}(\mathrm{H})$. Horizontal cracks were located at the mid-height of the specimen on the left, with a length of $100 \mathrm{~mm}$ and three different widths of $30 \mathrm{~mm}, 40$ $\mathrm{mm}$ and $50 \mathrm{~mm}$. The height (or thickness) of the specimen is shown in Figure 1.

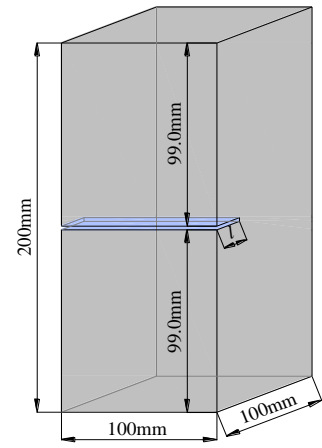

Fig. 1. Test Design (in mm)

\section{B. $\quad$ Test Method}

The testing instruments mainly included test loading system, acoustic emission monitoring system and a video camera. The loading system used was the RLJW-2000 servo controlled rock pressure testing system which uses piston driven by servo-controlled motor to pump high pressure oil into the loading oil tank for applying and controlling axial pressure (or displacement), with a maximum axial pressure $2,000 \mathrm{kN}$ and maximum axial displacement $100 \mathrm{~mm}$ at accuracy of $\pm 1 \%$. The test was loaded by controlling displacement at $0.003 \mathrm{~mm} / \mathrm{min}$.

The acoustic emission monitoring device used was MISTRAS series PCI-2 acoustic emission monitoring and analysis system which monitors crack propagation dynamically and in real time. The system has with a threshold of $40 \mathrm{~dB}$, floating threshold of $6 \mathrm{~dB}$ (when ambient noise is smaller than $20 \mathrm{~dB}$ ), and average wave speed of 2,286m /s and 6-channel location. Before testing, the specimen was underwent material acoustic characteristic matrix tests. During testing, the tip of the probe and the contact between the specimen and the pad were evenly applied with vaseline for reflection effect and its interference to sound signal emission, to ensure that the emitted sound signals can be well received by the sensor. The acoustic emission probes were mounted on the specimen with adhesive tape and the sensitivity test was carried out for the sensors before testing, to ensure that the signal magnitude captured by every probe is more than $90 \mathrm{~dB}$.

The main control and monitoring system for testing is shown as Figure 2. During testing, the loading system, the acoustic emission system and the camcorder were activated and synchronized for the same timing, in order to process data and analyze the closure, initiation and propagation of cracks.

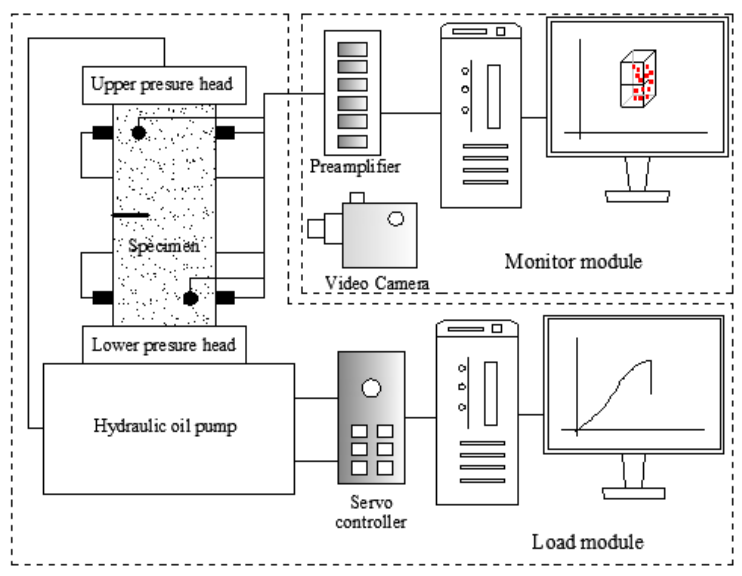

Fig. 2. Loading and Monitoring System for testing

\section{TEST RESULT ANALYSES}

\section{A. Analyzing Propagation characteristics of Surface Crack}

During loading, the tip of the open edge pre-crack had higher stress concentration than other parts of the specimen, so that the new crack started at the tip of the pre-existing crack. The propagation processes of various groups of surface cracks are shown in Figures 3-5, and the resulted crack types shown in Table 1.

TABLE I. CRACK TYPES IN SPECIMENS

\begin{tabular}{|c|c|c|}
\hline Crack number & Crack Type & Stress Level \\
\hline Pref1 & Pre-existing Horizontal Edge Crack & 0 \\
\hline Crack A1 & Wing Tension Crack & $12.5 \mathrm{MPa}$ \\
\hline Crack A2 & Anti-wing Tension Crack & $14.9 \mathrm{MPa}$ \\
\hline Crack A3 & Secondary Tension Crack & $14.9 \mathrm{MPa}$ \\
\hline Pref2 & Pre-existing Horizontal Edge Crack & 0 \\
\hline Crack B1 & Anti-wing Tension Crack & $2.5 \mathrm{MPa}$ \\
\hline Crack B2 & Secondary Tension Crack & $9.7 \mathrm{MPa}$ \\
\hline
\end{tabular}




\begin{tabular}{|c|c|c|}
\hline Pref3 & Pre-existing Horizontal Edge Crack & 0 \\
\hline Crack C1 & Wing Tension Crack & $3.6 \mathrm{MPa}$ \\
\hline Crack C2 & Anti-wing Tension Crack & $5.8 \mathrm{MPa}$ \\
\hline Crack C3 & Anti-wing Tension Crack & $6.3 \mathrm{MPa}$ \\
\hline Crack C4 & Secondary Tension Crack & $7.5 \mathrm{MPa}$ \\
\hline
\end{tabular}

- When crack width $l=30 \mathrm{~mm}$ as exemplified in Specimen 1-1, 1-2, 1-3, the propagation process is shown in Figure 3. Taking Specimen 1-1 as an example, the results of test are as follows.

At the early stage of loading, the pre-existing crack closed and its tip appeared scaling off under gradually increasing load. When the load increased to $12.5 \mathrm{MPa}$ or about $83.89 \%$ of peak strength, a macro-crack started from the tip of the pre-existing crack and propagated rapidly in an angle of $86^{\circ}$ from the horizontal direction to the upper end of the specimen, resulting in a wing tension crack $\mathrm{A} 1$. When the load reached to the specimen's peak strength of $14.9 \mathrm{MPa}$, an anti-wing tension crack A2 appeared from the tip of the preexisting crack. At the same time, a secondary tension crack A3 began from the lower face of the specimen. The specimen mainly suffered from tension failure.
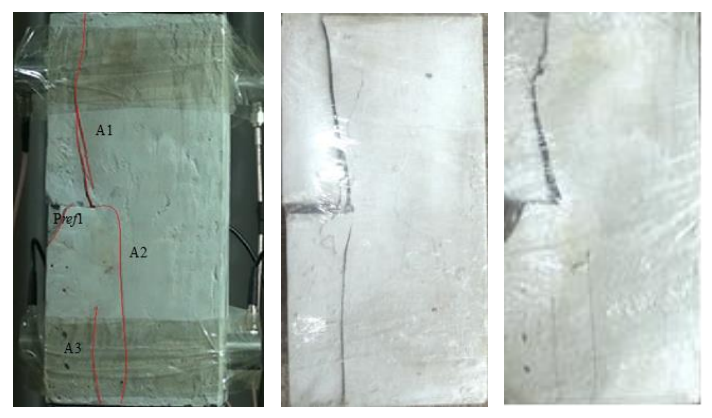

$\begin{array}{llll}\text { (a) Specimen 1-1 } & \text { (b) Specimen 1-2 } & \text { (c) Specimen 1-3 }\end{array}$

Fig. 3. Crack propagation process when crack width $l=30 \mathrm{~mm}$

- When crack width $l=40 \mathrm{~mm}$ as exemplified in Secimen 2-1, 2-2, 2-3, the propagation process is shown in Figure 4. Taking Specimen 2-1 as an example, the results of test are as follows.

When the load increased to $2.5 \mathrm{MPa}$ or about $25.77 \%$ of the peak strength, a Mode I crack started from the tip of the specimen and rapidly connected to its lower end of the specimen, forming an anti-wing tension crack B1, with the propagation direction perpendicular to the pre-existing crack. When the load reached to the peak strength of the specimen, a secondary tension crack B2 started from the tip of the preexisting crack, propagated in the loading direction and finally merged with the anti-wing tension crack B1, while the specimen totally disintegrated from tension failure.

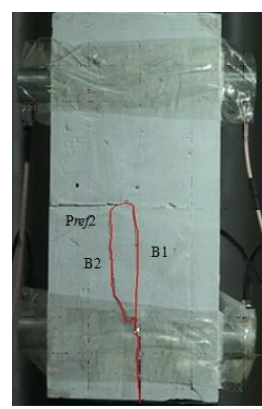

(a) Specimen 2-1

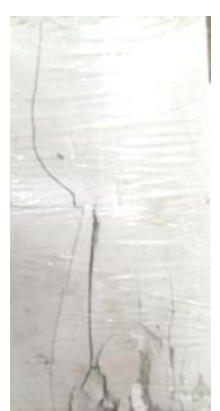

(b) Specimen 2-2

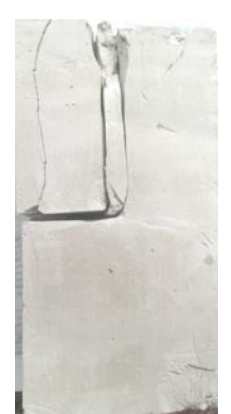

(c) Specimen 2-3
Fig. 4. Crack propagation process when crack width $l=40 \mathrm{~mm}$

- When crack width $l=50 \mathrm{~mm}$ as exemplified in specimen 3-1, 3-2, 3-3, the propagation process is shown as Figure 5. Taking Specimen 3-2 as an example, the results of test are as follows.

When the load increased to $3.6 \mathrm{MPa}$ or about $48 \%$ of the peak strength, a marco wing tension crack $\mathrm{C} 1$ developed from the tip of pre-existing horizontal edge crack, with an initiation angle and propagation angle of $78^{\circ}$ and $90^{\circ}$. When the load increased to $5.8 \mathrm{MPa}$ and $6.3 \mathrm{MPa}$, anti-wing tension crack $\mathrm{C} 2$ and $\mathrm{C} 3$ developed upward and downward from the tip of the pre-existing crack in an angle of $90^{\circ}$. When the load reached to the peak strength of $7.5 \mathrm{MPa}$, secondary tension crack $\mathrm{C} 4$ developed on the specimen and the specimen again was disintegrated by tension failure.

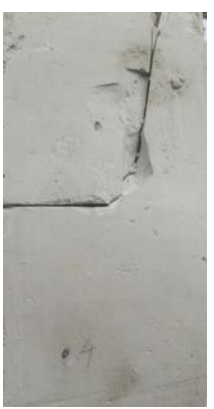

(a) Specimen 3-1

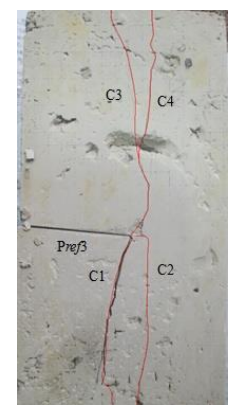

(b) Specimen 3-2

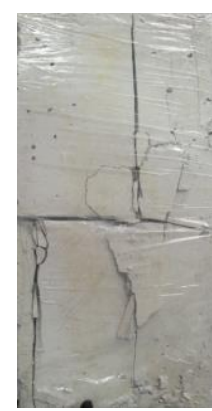

(c) Specimen 3-3
Fig. 5. Crack propagation process when crack width $l=50 \mathrm{~mm}$

Notes: the Pef1 Pef3 (Pre-existing fracture) are preexisting horizontal edge cracks with width respectively as $30 \mathrm{~mm}, 40 \mathrm{~mm}$ and $50 \mathrm{~mm}$ in figures $3-5$.

The crack initiation strength and uniaxial compressive strength of specimens are summarized in Table 2. According to Table 2, the crack initiation strength and average uniaxial compressive strength decrease with increasing width of the pre-existing horizontal edge crack. When their width increasing from $30 \mathrm{~mm}$ to $50 \mathrm{~mm}$, the average crack initiation strength reduces from $11.3 \mathrm{MPa}$ to $1.5 \mathrm{MPa}$ and the strength specimen peak strength ratio reduces from $14.2 \mathrm{MPa}$ to 7.6MPa. The ratio of crack initiation strength to peak strength reduces from 0.79 to 0.19 .

\section{B. Analysis of Acoustic Emission Characteristics}

When crack width $l=30 \mathrm{~mm}$, the typical AE results are exemplified by channel 1 in specimen $1-1$. The stress timeenergy and time-magnitude curves have been analyzed, with the results are shown in Fig. 6 (a). When the wing tension crack A1 started, the AE energy and the magnitude was 20,448 and $99 \mathrm{~dB}$ respectively, and the stress has a moderate but notable sudden drop. When the loading stress reached the peak strength of the specimen, anti-wing tension crack A2 and secondary tension crack A3 appeared, while the AE energy rapidly climbed to 34,528 and the magnitude became $>100 \mathrm{~dB}$ (note that a measuring range of set to 40 100 , hence magnitude greater than $100 \mathrm{~dB}$ was not reflected). When crack width $l=40 \mathrm{~mm}$ as represented by the channel 1 
in specimen 2-1, the stress-time-energy curve and the stresstime-magnitude curve have been analyzed, as shown in Figure 6 (b). When the wing tension crack B1 started, the energy value was 17,829 and the stress had a small sudden drop. When the loading time reached $1,576 \mathrm{~s}$, stress had a major drop, and the energy value jumped up to 59,535 , but no surface crack was observed. When stress reached the peak strength of $7.5 \mathrm{MPa}$ of the specimen, secondary tension crack B2 appeared, and the AE energy value climbed rapidly to 18,517 . When crack width $l=50 \mathrm{~mm}$ as exemplified by the channel 1 in specimen 3-2, the stress-time-energy curve and the stress-time-magnitude curve are shown in Figure 6 (c). When crack $\mathrm{C} 1$ initiated, the $\mathrm{AE}$ energy was respectively 11547 , and the stress began to fluctuate. With load increasing, crack C2 and C3 started, causing stress, AE energy and magnitude to change suddenly. When peak stress was applied on the specimen, secondary tension crack $\mathrm{C} 4$ started, causing the AE energy and the magnitude to abruptly change to 14,797 and $98 \mathrm{~dB}$ respectively.

TABLE II. CRACKING STRENGTH AND UNIAXIAL COMPRESSIVE STRENGTH

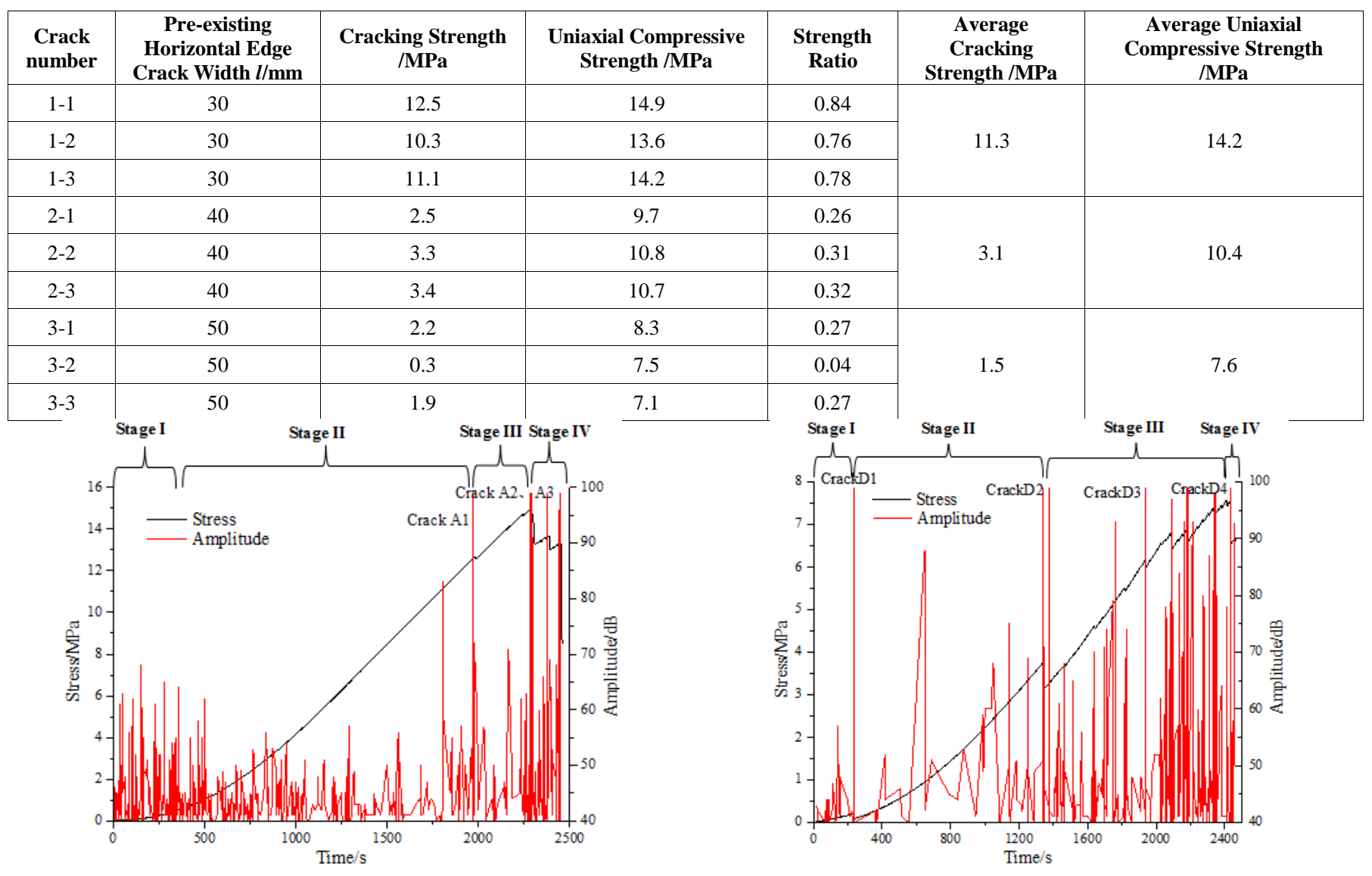

(a) Specimen1-1 $(l=30 \mathrm{~mm})$

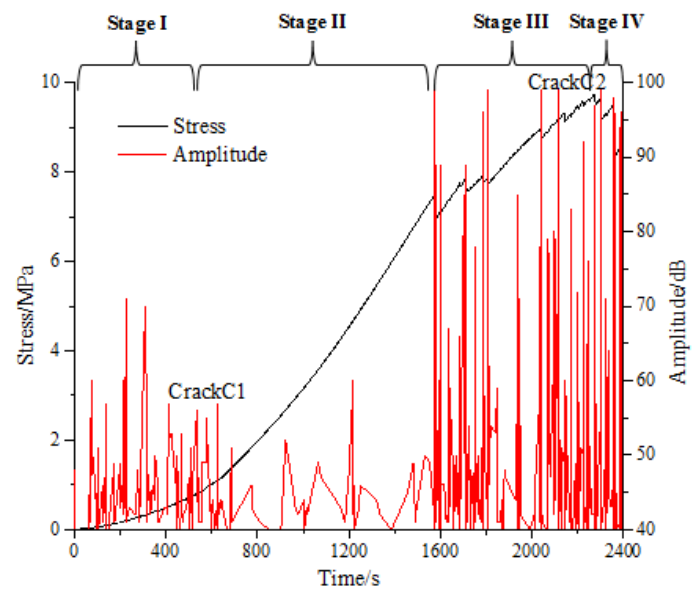

(b) Specimen 2-1 $(l=40 \mathrm{~mm})$ (c) Specimen 3-2 $(l=50 \mathrm{~mm})$

Fig. 6. Acoustic emission characteristic curves

As shown in Figure 6, the compression of the rock-like specimen with horizontal edge crack can be divided into four stages namely initial compaction stage (Stage I), elasticity stage (Stage II), macro extension of crack stage (Stage III) and specimen failure stage (Stage IV).

In summary, the following characteristics can be found for the crack propagation and its acoustic emission in the groups of specimens tested: (1) Wing tension crack firstly appeared in the specimen at the tip of the pre-existing crack, with an average cracking angle as $84.6^{\circ}$ from horizontal direction and propagation angle close to the loading direction. (2) With the width of the horizontal edge crack increasing, the uniaxial compressive strength and the crack initiation strength gradually decreased, the time of crack initiation was changed from the end of the elasticity stage to the end of the initial compaction stage, which is earlier. (3) At the period of stress not pulse, the acoustic emission signals from the 
specimen were weak, with lower AE energy value and magnitude, suggesting that there was no or only minor crack occurring within it. When the stress changed in "stepwise" manner, the specimen produced much stronger acoustic emission signals with rapidly increasing energy value and magnitude, indicating the development of macro crack. (4) When the rock-like materials with horizontal edge cracks were applied with uniaxial compression, primarily wing, anti-wing and secondary tension cracks started from the tip of the pre-existing crack, and eventually crack extended into the crack-free region and then connected to each other and joined the pre-existing crack, resulting in tensile fracture in "J" shape or "reverse $\mathrm{J}$ " shape and causing the specimen to fail for tension.

\section{CONCLUSIONS}

Under uniaxial compression, tension crack firstly appeared in the rock-like specimen with pre-existing horizontal edge crack at the tip of its pre-existing crack, with an average crack initation angle of $84.6^{\circ}$, and it propagated rapidly in an angle close to the loading direction to the upper and lower ends of the specimen. With the width of the preexisting crack increasing, the uniaxial compressive strength and the crack initiation strength of specimen decreased gradually. When the load reached the specimen's peak strength, anti-wing and secondary tension cracks started and crack extended into the crack-free region and then connected to the pre-existing crack, resulting in tensile fracture in "J" shape or "reverse $J$ " shape and causing the specimen to fail in tension. At the period of stress not pulse, the acoustic emission signals from the specimen were weak, with lower $\mathrm{AE}$ energy value and magnitude, suggesting that there was no or only minor crack occurring. When the stress changed in "stepwise" manner, the specimen produced stronger acoustic emission signals with rapidly increasing energy value and magnitude, indicating the development of macro crack.

\section{ACKNOWLEDGMENT}

The research described in this paper was financially supported by Taishan Scholar Talent Team Support Plan for Advantaged \& Unique Discipline Areas, National Science Foundation of China (No. 51428401, No. 51274135, No. 51574159), Shandong Provincial Natural Foundation (No. ZR2018LE008), Doctoral Research Foundation of Liaocheng University.

\section{REFERENCES}

[1] X Zhang, R. G. Jeffrey and B. S. Wu, "Mechanics of edge crack growth under transient pressure and temperature conditions," Int. J. Sol. Str., vol. S69-70, pp. 11-22, 2015.
[2] B. Lecampion, A. Bunger and X. Zhang, "Numerical methods for hydraulic fracture propagation: A review of recent trends," J. Nat. Gas Sci. Eng., vol.49, PP. 66-83, 2018.

[3] X. Zhang, B. S. Wu, R. G. Jeffrey, L. D. Connell and G. Q. Zhang, "A pseudo-3D model for hydraulic fracture growth in a layered rock,” Int. J. Sol. Str., vol.115-116, pp.208-223, 2017.

[4] B. Shen, J. Shi and N. Barton. "An approximate nonlinear modified Mohr-Coulomb shear strength criterion with critical state for intact rocks,” J. Rock Mech. Geol. Eng., vol.10, pp.828-835, 2018.

[5] D. W. Yin, S. J. Chen, X. Q. Liu , and H. F. Ma, "Effect of joint angle in coal on failure mechanical behavior of rock-coal combined body," Q. J. Eng. Geol. Hydroge., vol. 51, pp. 202-209, 2018.

[6] S. J. Chen, D. W. Yin, B. L. Zhang, H. F. Ma, and X. Q. Liu, "Study on mechanical characteristics and progressive failure mechanism of roof-coal pillar structure body," Chin J. Rock Mech. Eng., vol. 36, pp. 1588-1598, 2017.

[7] Z. F. Zhu, G. Q. Chen, H. Y. Xiao, H. Liu and C. Zhao, "Study on crack propagation of rock bridge based on multi parameters analysis of acoustic emission," Chin. J. Rock Mech. Eng., vol. 37, pp. 909918, 2018.

[8] Q. B. Zhang and J. Zhao, "Determination of mechanical properties and full-field strain measurements of rock material under dynamic loads,” Int. J. Rock Mech. Min. Sci., vol. 60, pp.423-439, 2013.

[9] C. Zhang, W. G. Cao, Z. Xu and M. He, "Initial macro-deformation simulation and determination method of micro-crack closure stress for rock," Rock Soil mech., vol. 39, pp. 1281-1289, 2018.

[10] W. G. Cao, H. Zhao and L. Zhang, "Damage statistical softening constitutive model for rock considering effect of damage threshold and its parameters determination method," Chin. J. Rock Mech. Eng., vol. 27, pp. 1148-1154, 2008.

[11] J. Peng, M. Cai, G. Rong, et al. "Stresses for crack closure and its application to assessing stress-induced microcrack damage," Chin. J. Rock Mech. Eng., vol. 34, pp. 1091-1100, 2015.

[12] W. T. Liu and J. J. Shen, "Experimental study of propagation mode of crack in real rock specimens with a single crack," Chin. J. Rock Mech. Eng., vol. 35, pp. 1182-1189, 2016.

[13] F. N. Dang, J. Y. Fang and W. H. Ding, "Fractal comparison research of concrete samples under static under static and dynamic uniaxial tensile using CT," Chin. J. Rock Mech. Eng., vol. 34, pp. 2922-2928, 2015.

[14] X. Z. Sun, B. Shen and B. L. Zhang, "Experimental study on propagation behavior of three-dimensional cracks influenced by intermediate principal stress," Geomech. Eng., vol. 14, pp. 195-202, 2018.

[15] T. S. Yun, Y. J. Jeong, K. Y. Kim and K. B. Min, "Evaluation of rock anisotropy using 3D X-ray computed tomography," Eng. Geol., vol. 163, pp. 11-19, 2012.

[16] B. Shen, J. Shi, M. Rinne, et al, "Two-dimensional displacement discontinuity method for transversely isotropic materials," Int. J. Rock Mech. Min. Sci., vol. 83, pp. 218-230, 2016.

[17] B. Shen, T. Siren and M. Rinne, "Modelling Fracture Propagation in Anisotropic Rock Mass," Rock Mech. Rock Eng., vol. 48, pp. 1067-1081, 2015.

[18] B. Shen, Y.B. Jung, E.S. Park, and T. K. Kim, "Modelling the effect of ice swelling in the rock mass around an LNG underground storage cavern using FRACOD,” Geo. Eng., vol.18, pp. 1-18, 2015.

[19] S. Zhang, Y. Q. Lu and Q. Z. Wang, "Measurement of dynamic fracture propagation toughness of rock and observation of dynamic arrest phenomenon using P-CCNBD specimens," Rock Soil Mech., vol. 38, pp. 3095-3105, 2017. 